\title{
Heart rate changes accompanying differential classical conditioning of somatic response systems in the rabbit
}

\author{
D. A. POWELL and MARK LIPKIN \\ Neuroscience Laboratory, Veterans Administration Hospital, Columbia, South Carolina 29201
}

\begin{abstract}
Leg flexion and head turning were elicited by footshock paired with tone CSs. The original HR CR was a deceleration in rate. However, this deceleration developed a later occurring accelerative component during the middle and late stages of acquisition. These data, combined with prior research, suggest that the early decelerative $\mathrm{CR}$ is associated with behavioral freezing and attention while the later accelerative component is associated with the acquisition of somato-motor CR.s in response to the CS-UCS contingency.
\end{abstract}

The classically conditioned heart rate (HR) response accompanying coreneoretinal potential (CRP) conditioning is a deceleration during early acquisition sessions (Schneiderman, 1970). However, it becomes biphasic during later sessions consisting of an initial brief deceleration followed by a longer lasting acceleration (Powell, Lipkin, \& Milligan, in press). Several stimulus and organismic variables have been related to these changes (Powell et al., in press; Powell \& Joseph, in press). In the present experiment these findings were generalized to a situation in which ditterent somatic responses, viz., leg flexion (LF) and head turning (HT), were assessed and in which the shock UCS was administered to a different location, viz., foot, rather than the eyelids as is necessary in CRP conditioning.

\section{METHOD}

\section{Subjects}

Twelve New Zealand albino male and female rabbits were used as subjects. The animals were maintained on ad-lib food and water and on a 7:00 a.m.-7:00 p.m. light-dark cycle. All subjects were run during the daylight portion of this cycle.

\section{Apparatus}

The animals were run in a canvas sling with four holes in the bottom through which protruded its front and back legs. This sling was attached with snaps to the tov of the experimental chamber, deactivated refrigerator shell. The subject was thus restrained so that it could not make larger locomotor responses but was able to move its front and hind limbs. The shock electrodes consisted of stainless steel wire taped to the back left paw of the animal. The hair on the paw was removed with a depilator before the electrodes were situated. Seventy dB SPL white noise and $80 \mathrm{~dB}$ SPL CSs were presented through 12-in speakers situated $30 \mathrm{~cm}$ above the subjects head. The HR and LF responses were recorded on a Gilson (GME) polygraph. The HR electrodes consisted of stainless steel safety pins inserted into the left back haunch and the right front leg. These electrodes

This research was supported by VA Institutional Research Funds Project 5737-02. Requests for reprints should be sent to D. A. Powell, Neuroscience Laboratory, Veterans Administration Hospital, Columbia, South Carolina 29201. resulted in no observable discomfort or infection. The shock source was a BRS Model SC-001 shock generator. The CSs consisted of tones produced by Bud code practice oscillators. The recording and control equipment was located in a room adjacent to the experimental chamber. Electronic timers in conjunction with BRS solid state programming equipment automatically programmed the delivery of the shock, the CS-US intervals, etc.

\section{Procedure}

The subjects received a differential classical conditioning paradigm in which a $304-$ or $1216-\mathrm{Hz} 1-\mathrm{sec}$ tone served as $\mathrm{CS}+$ or $\mathrm{CS}-$. The CS+ was always reinforced with a .5-sec, 3-mA electric shock through the shock electrodes; $\mathrm{CS}-$ was never reinforced with shock. The LF response was measured by EMG electrodes which were attached to the thigh of the left foreleg and led to the Gilson polygraph as described above. In addition to measuring the LF and CR, an orienting type response was also observed by visually observing the animal through a one-way mirror. This response consisted of an abrupt tensing of the head, neck, and ears, as well as turning of the head toward the source of the CS. The term orienting, as used here, is descriptive only and is not used in a theoretical or conceptual sense as, for example, was done by Graham and Clifton (1966). Thus, to avoid confusion, this response will hereafter be referred to as the "head turning" (HT) response. The presence or absence of both LF and HT CRs was recorded during each CS on alternate trials. The intertrial interval was $90 \mathrm{sec}$. Every eighth trial was a nonreinforced test trial (half were CS+ and half $\mathrm{CS}_{-}$) during which HR CRs were assessed. These responses were measured as percent change scores based on (a) a baseline measure consisting of the mean duration of the 10 beats occurring immediately before CS onset, and (b) two 10-beat post-CS measures consisting of the duration of two successive blocks of ten beats after the onset of the CS. The percent change score was defined as the post-HR score minus the prescore divided by the prescore. Each subject received 2 days adaptation in which the tones were presented alone (viz., not followed by the UCS). These adaptation sessions were followed by 20 days of acquisition in which the iones were reinforced with shock as described above.

\section{RESULTS}

The results are shown in Figure 1. This graph shows LF and HT CRs at the top while HR CRs are shown in the lower portion. HR changes are shown only for the 

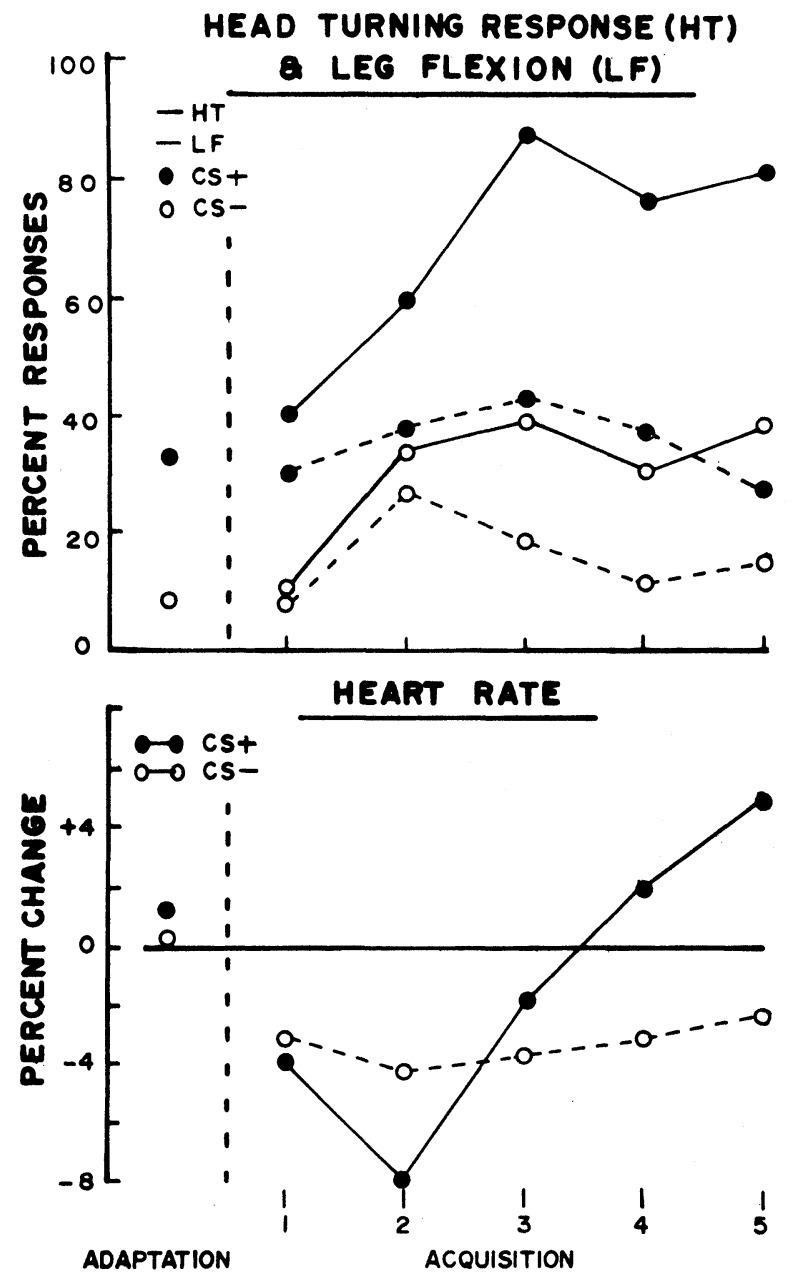

BLOCKS OF SESSIONS

Figure 1. Heart rate (bottom) and somatic (top) CRs in response to a 1-sec CS+ reinforced with shock and a CS- never reinforced with shock. Data are shown for one block of two adaptation sessions, and five blocks of four acquisition sessions. The HR CRs represent the mean percent change from a pre-CS baseline of the second ten heart beats following CS onset. The HT response (solid line) and leg flexion CRs (dotted line) were assessed only during the 1-sec tone CS.

second block of posttone beats since prior research (Powell et al, in press) has shown that the accelerative HR component does not become manifest until this time. Looking first at the HR changes associated with CS+ and CS-, it can be seen that, during the first two blocks of four acquisition sessions, there were greater HR decelerations to CS+ than to CS-. Repeated measures analysis of variance of the HR data showed the overall difference between $\mathrm{CS}+$ and $\mathrm{CS}-$ to be significant $(\mathrm{F}=6.41, \mathrm{df}=1 / 11, \mathrm{p}<.05)$, as was the interaction of the CS+ $-\mathrm{CS}-$ dimension and sessions $(\mathrm{F}$ $=13.86, \mathrm{df}=1 / 44, \mathrm{p}<.01)$. HR CRs during the latter portions of the experiment were relatively more accelerative to $\mathrm{CS}+$ than to $\mathrm{CS}$ - and actually crossed zero during the latter portions of the experiment to become accelerative changes of from $3 \%$ to $4 \%$, whereas CS- changes averaged about $2 \%$ decreases in rate. The significant interaction of the CS+ - CS-dimension and sessions suggests that this change in HR CR topography over sessions was reliable.

Reference to the top portion of Figure 1 shows that discriminative changes occurred in the leg flexion as well as the "head turning" response systems. The HT response (solid lines) showed significantly more CRs to $\mathrm{CS}+$ than $\mathrm{CS}-(\mathrm{F}=16.67, \mathrm{df}=1 / 44, \mathrm{p}<.01)$, and attained maximum frequency around the middle of the experiment. Analysis of variance of the LF responses showed that significantly more responses occurred to the $\mathrm{CS}+$ than $\mathrm{CS}-(\mathrm{F}=5.03$, df $=1 / 11, \mathrm{p}<.05)$, but the overall magnitude of responding in the LF response system was considerably less than that in the HT system. Moreover, LF responses appeared to decline during the latter portions of the experiment as compared to the HT responses, which maintained an asymptotic level around $80 \%-90 \%$. These data thus parallel those of previous experiments in which the corneoretinal potential response was assessed; discriminative responding was obtained in both the HT and the LF response systems as a function of acquisition sessions and, moreover, the acquisition of these somatic CRs was related to changes in the HR CR. As the latter was changing from a decelerative response to a relatively large acceleration, HT and LF discrimination occurred.

\section{DISCUSSION}

The results of the present experiment support those of previous studies which have shown that early during classical conditioning of somatic response systems in the rabbit, the cardiovascular system exhibits cardiac slowing. On the other hand, as the animal reaches asymptotic responding in somatic systems, the HR CRs become accelerative. The results of several experiments suggest that HR conditioning is decelerative early during training because motor inhibition or behavioral freezing is maximal during this time. HR increases then occur later during acquisition as behavioral freezing is reduced. Bruner (1969), for example, used electric shock as the UCS in cats and obtained decelerative HR CRs accompanied by pronounced inhibition of tremor responding. Obrist and associates have also related HR decreases to inhibition of motor responding in dogs (Obrist, Webb, Sutterer, \& Howard, 1970). It has been shown that EMG responses, as well as hippocampal theta activity, increase during acquistion of the CRP response in the rabbit (Powell \& Joseph, in press). Moreover, as these increases in skeletal responding occurred, the accelerative component of the HR CR became more pronounced.

The earlier occurring deceleration HR CRs are also compatible with other interpretations of cardiac-somatic relationships. Lacey and Lacey (1971), for example, have suggested that decelerative HR CRs are associated with attentional mechanisms indicative of sensory processing, while accelerative CRs are associated with response execution. Similarly, Graham and Clifton (1966) maintain that HR decreases are associated with the "orienting reflex" originally studied by Sokolov (1963), while HR accelerations are associated with the "defensive 
reflex." The early occurring decelerative HR CRs and later appearing accelerative CRs of the present study may obviously be interpreted within either of these theoretical frameworks.

\section{REFERENCES}

Bruner, A. Reinforcement strength in classical conditioning of leg flexion, freezing, and heart rate in cats. Conditioned Reflex, 1969, 4, 24-31.

Graham, F. K., \& Clifton, R. K. Heart rate change as a component of the orienting response. Psychological Bulletin, 1966, 65, 305-320.

Lacey, J. I., \& Lacey, B. C. Some autonomic-central nervous system interrelationships. In: P. Black (Ed.). Physiological correlates of emotion. New York: Academic Press, 1971. 205-227.
Obrist, P. A., Webb, R。A., Sutterer, J. R。, \& Howard, J. L. The cardiacsomatic relationship: Some reformulations. Psychophysiology, 1970, 6, 569-587.

Powell, D. A., \& Joseph, J. A. Autonomic-somatic interaction and hippocampal theta activity. Journal of Comparative and Physiological Psychology, in press.

Powell, D. A., Lipkin, M., \& Milligan, W. L. Concomitant changes in classically conditioned heart rate and corneoretinal potential discrimination in the rabbit (Oryctolagus Cuniculus). Learning and Motivation, in press.

Schneiderman, N. Determinants of heart rate classical conditioning. In: S. Reynierse (Ed.), Current issues in animal learning. Lincoln: University of Nebraska Press, 1970.

Sokolov, E. N. Perception and the conditioned reflex. New York: MacMillan, 1963

(Received for publication September 19, 1974.)

Bulletin of the Psychonomic Society

1975, Vol. 5 (1), 30-32

\title{
Rejection of input in the processing of an emotional film*
}

\author{
PETER SUEDFELD \\ The University of British Columbia, Vancouver, British Columbia, Canada \\ MATTHEW HUGH ERDELYI \\ Brooklyn College of The City University of New York, \\ New York, New York 11210 \\ and \\ CAROLYN R. CORCORAN \\ Douglass College, Rutgers - The State University, \\ New Brunswick, New Jersey 08903
}

\begin{abstract}
Subjects saw a film sequence that was abruptly terminated and followed by a letter matrix. One group saw a film of high emotionality (HE) while a second group saw one of low emotionality (LE). A control group was shown a blank white slide (B) followed by the same letter matrix as seen by the other subjects. ANOVA showed that control subjects had the highest sensitivity for the letter matrix and the $\mathrm{HE}$ group had the lowest sensitivity; the difference between $\mathrm{HE}$ and LE was not significant. When film subjects were divided into those who rated the films as high disgusting (HD) and those who rated the film as low disgusting (LD), ANOVA showed that control subjects had the highest sensitivity, LD subjects an intermediate level, and HD subjects the lowest, the difference between HD and LD in this case being significant. Results were interpreted as representing emotion induced rejection of input.
\end{abstract}

Recent research has demonstrated that emotional stimuli, whether verbal or pictorial, can produce processing disruptions for other proximate stimuli (Erdelyi \& Appelbaum, 1973; Erdelyi \& Blumenthal,

*This research was financed by grants from the Rutgers University Research Council to the first two authors. We are grateful to Mrs. Boston, Metuchen Chapter, American Cancer Society, for providing the film used in the study, and to S. Coren and $L$. M. Ward for their comments on an earlier version of the manuscript.
1973; Schultz, 1971; Tulving, 1969). "Proximate" can be defined either spatially or temporally, depending upon whether simultaneous or sequential presentation is used.

As a general rule, such experiments present discrete stimuli for brief durations. Because this is a somewhat artificial procedure, it is open to the criticism of questionable generalizability or external validity. Perception in the nonlaboratory environment usually involves the continuous processing of complex inputs 